\section{Nutrición perioperatoria en protocolos quirúrgicos para una mejor recuperación postoperatoria (Protocolo ERAS)}

\author{
ANDRÉS SÁNCHEZ C. ${ }^{1}$, KARIN PAPAPIETRO V. ${ }^{2}$
}

\section{Perioperative nutrition in ERAS Protocols}

The trauma involved in any surgical procedure, even if elective, causes a metabolic stress response characterized by postoperative insulin resistance (PIR). PIR is considered a surgical stress marker and is associated with increased morbidity and postoperative length of stay. PIR worsens when the patient is operated in a state of prolonged preoperative fasting or when postoperative feeding is delayed. The ERAS Protocols (Enhanced Recovery After Surgery) includes anesthetic, surgical, kinesiology, nutritional and nursing interventions aimed to modulate PIR. The nutritional perioperative interventions in the ERAS protocols, focus on avoiding prolonged preoperative fasting by oral carbohydrate loading up to two hours before surgery, accompanied by early postoperative feeding through the digestive tube. These nutritional perioperative interventions are safe and effective to reduce complications and postoperative stay, even in patients with well controlled type 2 diabetes. Nevertheless, their implementation and compliance are rather low, therefore, we must continue to make efforts in order to change perioperative nutritional management of our patients to achieve the best possible postoperative recovery.

(Rev Med Chile 2017; 145: 1447-1453)

Key words: Carbohydrate Metabolism; Diet, Carbohydrate Loading; Insulin Resistance; Nutrition Therapy; Perioperative Care; Perioperative Period.

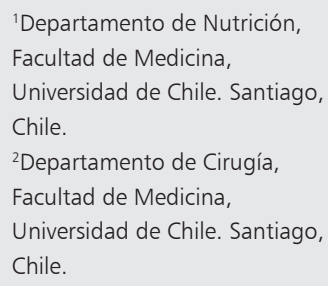

No hubo apoyo financiero para la elaboración de este manuscrito. Autores no declaran conflicto de intereses.

Recibido el 11 de mayo de 2016 , aceptado el 4 de enero de 2017.

Correspondencia a: Andrés Sánchez C. Independencia 1027. Departamento de Nutrición, piso 2, block K, Facultad de Medicina, Universidad de Chile. andres.sanchez.cordoba@gmail. com

\section{Ayuno preoperatorio prolongado, respuesta metabólica al estrés y resistencia a la insulina postoperatoria}

$\mathrm{E}$ 1 número de cirugías a nivel mundial es muy alto y genera grandes costos económicos a cualquier sistema de salud ${ }^{1}$. Chile no es la excepción y se estima que anualmente se realizan más de 56.490 cirugías digestivas electivas, con costos que varían entre miles y millones de pesos, sin incluir los costos de las complicaciones médicas o quirúrgicas que se pudieran presentar ${ }^{2,3}$.

Las complicaciones, estadía hospitalaria y mortalidad en postcirugía dependen de múltiples factores, como el tipo de cirugía (digestiva alta, colorrectal, torácica, traumatológica, ginecológica, etc), vía de abordaje abierto vs laparoscópico, estado nutricional y comorbilidades del paciente, etc. Por ejemplo, en Estados Unidos de Norteamérica se ha reportado que en hemicolectomías por cáncer de colon las complicaciones y mortalidad son de $27,55 \%$ y $1,43 \%$, respectivamente, con una estadía hospitalaria promedio de 7,37 días ${ }^{4}$. Diversos estudios en Chile han reportado que la cirugía laparoscópica por cáncer colorrectal tiene en promedio una morbilidad de $25 \%$, mortalidad de $0,76 \%$ y estadía hospitalaria con mediana de 5 días ${ }^{5}$, cirugías por cáncer de recto tienen morbilidad y mortalidad de $13,6 \%$ y $0,3 \%$, respectivamente $^{6}$, y para esofagectomía, las complicaciones 
médicas van de 24 a 29,8\%, complicaciones quirúrgicas de 22,1 a $37,1 \%$ y tasas de mortalidad de 2,1 hasta $8,6 \%{ }^{7}$.

Datos como estos muestran que, al igual que sucede en otros países, en Chile los procedimientos quirúrgicos se asocian a un alto consumo de recursos en salud y, por lo tanto, es primordial el desarrollo de protocolos quirúrgicos que busquen resultados postoperatorios óptimos de la manera más eficiente posible.

Cualquier cirugía, aun si es electiva, constituye un traumatismo para el paciente y desencadena una respuesta refleja automática mediada por el sistema nervioso autónomo (simpático) y por el sistema endocrino (eje hipotálamo-hipófisis-glándula suprarrenal) aumentando la producción de catecolaminas y glucocorticoides, respectivamente. Esta respuesta integrada y coordinada se conoce como respuesta metabólica al estrés y se caracteriza por modificaciones en el tono cardiovascular, patrón respiratorio, disfunción gastrointestinal, depresión inmune, reacción inflamatoria, catabolismo proteico y alteraciones del metabolismo intermediario que llevan a un estado de resistencia a la insulina postoperatoria (RIP) e hiperglicemia ${ }^{8}$.

Si bien es cierto que la RIP es una condición metabólica que se presenta como respuesta al estrés después de cualquier procedimiento quirúrgico, estudios en voluntarios sanos han demostrado que la resistencia a la insulina también se desencadena por estados de ayuno prolongado9. Por lo tanto, si un paciente es llevado a cirugía en un estado de ayuno de más de 16 h, será operado bajo un estado de resistencia a la insulina preoperatoria que empeorará la hiperglicemia postcirugía.

Hoy en día, la RIP se entiende como una alteración del metabolismo intermediario, que se presenta secundaria al ayuno preoperatorio prolongado y a la respuesta metabólica al estrés estableciéndose como un marcador de estrés quirúrgico que es directamente proporcional a la magnitud de la cirugía ${ }^{10}$, causante del incremento de la morbilidad postoperatoria ${ }^{11} \mathrm{y}$ del aumento de la estadía hospitalaria ${ }^{12}$. Por tanto, se han ideado nuevos protocolos quirúrgicos que buscan modular esta condición.

\section{Protocolo ERAS}

En el año 2001, un grupo de cirujanos y anestesiólogos de Europa conformaron el Grupo de Estudio ERAS para la Mejor Recuperación Postoperatoria, por sus siglas en inglés (Enhanced Recovery After Surgery). Con base en una revisión sistemática de la literatura para identificar los cuidados perioperatorios óptimos en cirugía colorrectal, desarrollaron un plan de cuidados perioperatorios al que denominaron Protocolo ERAS, destinado a disminuir el estrés quirúrgico y acelerar la recuperación postoperatoria en un contexto de seguridad para el paciente. Publicado en 2005, constituyó el primer consenso internacional sobre cuidados perioperatorios para candidatos a resección colónica, incorporando 20 intervenciones pre, intra y postoperatorias multidisciplinarias de tipo quirúrgico, anestésico, de enfermería, kinésico y nutricional, siendo la cirugía mínimamente invasiva, la analgesia multimodal y las intervenciones nutricionales las 3 piedras angulares del Protocolo ERAS ${ }^{13}$ (Figura 1).

Durante el año 2010 el Grupo de Estudio ERAS se convirtió en una sociedad intercontinental (Sociedad ERAS) que actualiza, adapta y publica Protocolos ERAS y guías de cuidados perioperatorios para diversas cirugías entre ellas: cirugía colónica ${ }^{14}$, pélvica/rectal ${ }^{15}$, gastrectomía ${ }^{16}$, pancreatoduodenectomía ${ }^{17}$, cistectomía radical ${ }^{18}$, etc.

\section{Intervenciones nutricionales perioperatorias en el Protocolo ERAS}

\section{Prevención del ayuno preoperatorio prolongado utilizando carga de hidratos de carbono por vía oral}

Dado que uno de los desencadenantes de la RIP es el ayuno preoperatorio prolongado (APP), en las últimas 2 décadas se han desarrollado estudios que exploran la posibilidad de alimentar al paciente pocas horas antes de la cirugía, evitando el ayuno prolongado y operándolo en estado postprandial que disminuya la RIP después del estrés quirúrgico ${ }^{19}$.

El APP desde la media noche antes de una cirugía electiva ha sido uno de los "dogmas" practicados y transmitidos por generaciones de cirujanos y anestesiólogos, buscando minimizar el riesgo de broncoaspiración en el momento de la inducción anestésica e intubación. Sin embargo, a pesar que esta práctica es rutinaria en numerosas instituciones, no cuenta con evidencia científica que la sustente. Una completa revisión de las guías 


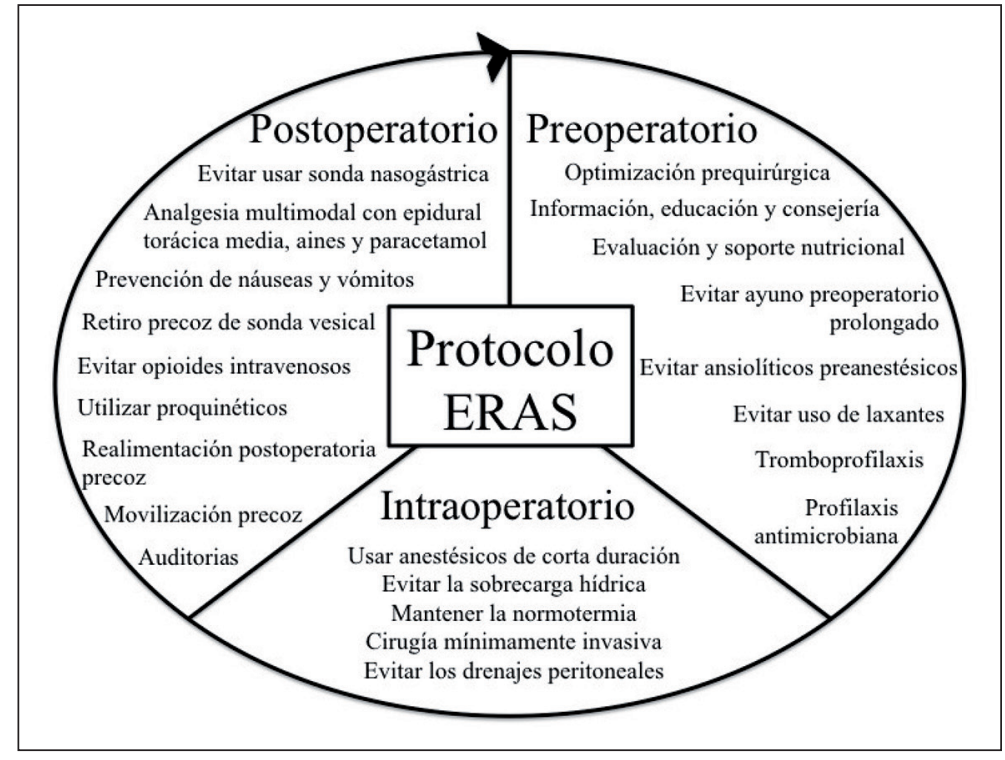

Figura 1. Intervenciones multidisciplinarias pre, intra y postoperatorias en el Protocolo ERAS. ERAS: enhanced recovery after surgery. Imagen adaptada de ERAS Society. de ayuno preoperatorio de diversas sociedades de anestesiología a nivel mundial, elaborada por O. Lungqvist y E. Soride, además de un metaanálisis Cochrane que incluyó 22 ensayos clínicos aleatorizados, ambos publicados en 2003, demostraron que evitar el APP con la administración de líquidos claros (té, jugos de fruta sin pulpa, agua con maltodextrina) hasta $2 \mathrm{~h}$ antes de la cirugía no aumentó la prevalencia de complicaciones e incluso disminuyó el volumen del contenido gástrico, en comparación con los pacientes operados con el ayuno tradicional ${ }^{20,21}$. Además, esta práctica ha demostrado reducir la sed, el hambre y la ansiedad previo a la cirugía ${ }^{22} y$, más importante aun, reducir la resistencia a la insulina ${ }^{23}$, la pérdida de nitrógeno y proteínas corporales ${ }^{24}$, manteniendo la fuerza ${ }^{25} \mathrm{y}$ conservando la masa magra en el postoperatorio ${ }^{26}$. Por lo tanto, el Protocolo ERAS indica que se debe permitir la ingesta de alimentos sólidos como un régimen liviano hasta $6 \mathrm{~h}$ antes de cirugía y la ingesta de té, café, jugo de frutas sin pulpa hasta $2 \mathrm{~h}$ antes de cirugía, recomendación que actualmente es avalada por múltiples sociedades europeas de Anestesiología y por la Sociedad Americana de Anestesiología ${ }^{27-29}$.

En el protocolo se ha estandarizado la administración rutinaria de $400 \mathrm{ml}$ de agua con maltodextrina al $12,5 \%$, por vía oral, hasta $2 \mathrm{~h}$ antes de cirugía electiva en todo paciente que no presente riesgo de vaciamiento gástrico retardado, por ejemplo, gastroparesia diabética.

\section{Carga de hidratos de carbono preoperatoria vía oral en pacientes con diabetes mellitus tipo 2}

Existen 2 publicaciones que avalan el uso de cargas de hidratos de carbono por vía oral en pacientes diabéticos tipo 2. En la primera, Breuer y cols., en 2006, evaluaron 188 pacientes diabéticos tipo 2 no insulino requirentes, sin complicaciones derivadas de la diabetes mellitus, ASA III-IV, candidatos a cirugía cardíaca electiva. En estos pacientes se suministró la carga de hidratos de carbono por vía oral estándar (maltodextrina 12,5\%) y se encontró que no hubo retraso en el vaciamiento gástrico ${ }^{30}$. Por su parte, Gustafsson y cols., en 2008, compararon el vaciamiento gástrico y las concentraciones de glucosa plasmática por $3 \mathrm{~h}$ después de una carga de hidratos de carbono en 3 grupos de pacientes: diabéticos tratados con dieta o hipoglicemiantes orales, diabéticos insulino requirentes y sujetos sanos ${ }^{31}$. Encontraron que los diabéticos bien controlados $(\mathrm{HbAlc}<7 \%)$ no tuvieron retraso en el vaciamiento gástrico ni alteraciones del control glicémico, en comparación con el grupo de sujetos sanos. Con base en estos hallazgos, el Protocolo ERAS actualmente considera que es seguro administrar maltodextrina al 12,5\% 2 a 3 h antes de cirugía en sujetos diabéticos tipo 2 bien controlados. 


\section{Realimentación postoperatoria precoz}

El manejo tradicional de la nutrición postoperatoria en cirugía digestiva, especialmente cuando se ha dejado una anastomosis, se ha caracterizado por mantener al paciente en ayuno, con sonda nasogástrica para evitar vómitos y prevenir el aumento de presión sobre la zona anastomótica y realizar el aporte nutricional a través de soluciones glucosadas intravenosas hasta que se resuelva espontáneamente el íleo postoperatorio (manifestado clínicamente por el retorno de ruidos hidroaéreos y paso de gases o deposiciones por ano). Una vez recuperada la función del tracto gastrointestinal, los cuidados tradicionales inician lentamente la administración de líquidos con escaso contenido de nutrientes (agua, te, gelatinas, etc.) y, según la tolerancia del paciente, progresan en consistencia hasta llegar a una alimentación sólida. Este manejo nutricional en el postoperatorio se ha practicado de manera rutinaria por años, bajo la creencia de que reduce las náuseas, vómitos, neumonía aspirativa y la dehiscencia de las anastomosis, pero también sin evidencia científica que avale esta práctica. Sin embargo, en la literatura actual, un creciente número de ensayos clínicos aleatorizados y metaanálisis han demostrado que la realimentación precoz por vía digestiva, desde el primer día postoperatorio, no se asocia a eventos adversos $y$, por el contrario, puede disminuir las infecciones postoperatorias, estadía hospitalaria y mortalidad ${ }^{32,33}$. Un metaanálisis publicado por Osland y cols. en 2011, que incluyó 15 ensayos clínicos aleatorizados con 1.240 pacientes de cirugía digestiva con anastomosis, demostró que la realimentación precoz administrada desde el primer día postoperatorio y proximal a la anastomosis, disminuye significativamente las complicaciones postoperatorias ${ }^{34}$.

Es frecuente que el paciente candidato a cirugía digestiva se encuentre desnutrido, lo cual es un factor de riesgo para mala cicatrización de las heridas y aumento en la morbimortalidad postoperatoria. Estudios en animales y humanos han demostrado que prolongar el ayuno durante el postoperatorio disminuye el contenido de colágeno de las anastomosis y la calidad de la cicatrización ${ }^{35}$, mientras que la realimentación precoz mantiene el trofismo intestinal e incrementa el contenido de colágeno y la fuerza de la cicatriz anastomótica ${ }^{36}$. A la fecha, ningún estudio o metaanálisis ha demostrado que la realimentación precoz con alimentos entregados proximal o distal a la anastomosis, aumente el riesgo de dehiscencia de sutura, por el contrario, existe una tendencia a disminuirla.

El régimen hídrico y los líquidos claros se han establecido clásicamente como el primer alimento para reiniciar la ingesta oral postoperatoria en los cuidados tradicionales. Sin embargo, actualmente no existe sustento científico que avale esta conducta, además de que su aporte nutricional es prácticamente nulo $y$, por lo tanto, no ofrecen ningún beneficio para controlar la resistencia a la insulina y el catabolismo proteico que se presenta durante el postoperatorio. Lo anterior hace suponer que la realimentación con agua y líquidos claros sin calorías retrasa el aporte de nutrientes y, por tanto, no modula la respuesta metabólica al estrés quirúrgico.

En las últimas décadas, estudios han demostrado que los pacientes quirúrgicos pueden tolerar la realimentación precoz con líquidos enriquecidos con hidratos de carbono, suplementos nutricionales orales (SNO), fórmulas enterales o directamente con alimentos sólidos. Soop y cols. demostraron que la realimentación precoz, con nutrición enteral vía sonda nasoyeyunal en cirugía colorrectal, logró controlar la resistencia a la insulina postoperatoria y mantener el balance nitrogenado ${ }^{37}$. Por lo tanto, se considera que realimentar precozmente al paciente con líquidos o sólidos que provean un adecuado aporte de nutrientes impacta positivamente, reduciendo el déficit calórico y modulando la respuesta metabólica al estrés en los días inmediatamente posteriores a la cirugía.

Un aspecto que es clave dentro del Protocolo ERAS en cirugía abierta y laparoscópica es el control estricto de la volemia durante el intra y postoperatorio. La sobrecarga hídrica del paciente (objetivado por aumento de hasta $3 \mathrm{~kg}$ de peso en el postoperatorio), produce edema de asas intestinales, retardo del vaciamiento gástrico, favorece el íleo postoperatorio y se asocia a más complicaciones, retrasando el alta hospitalaria ${ }^{38,39}$. Por lo tanto, evitar la sobrecarga hídrica es fundamental para favorecer la realimentación precoz del paciente en el postoperatorio.

Actualmente, el Protocolo ERAS propone que si el paciente ha recibido un manejo perioperatorio multimodal, con carga de hidratos de carbono preoperatorios, control óptimo del dolor, restricción de sobrecarga hídrica intravenosa, 
prevención de náuseas y vómitos, movilización precoz, etc. puede empezar a recibir líquidos con SNO el mismo día quirúrgico y un régimen de alimentos sólidos con 1.200-1.500 kcal a partir del primer día postoperatorio ${ }^{14}$. Recientemente se ha empezado a estudiar el uso de SNO enriquecidos con inmunonutrientes (glutamina, arginina, omega 3 y nucleótidos) en el postoperatorio pre$\mathrm{COz}$ de pacientes quirúrgicos y desnutridos, sin embargo, la evidencia aún es débil en cuanto a la efectividad de estos suplementos dentro de los Protocolos ERAS ${ }^{40}$.

\section{Efectividad del Protocolo ERAS}

Múltiples estudios en cirugía colorrectal, han demostrado que aplicando el Protocolo ERAS, se logra acelerar la recuperación del paciente y disminuir la estadía hospitalaria en comparación con los cuidados tradicionales ${ }^{41}$. Tres metaanálisis que incluyeron a los estudios de mejor calidad para evaluar el impacto del Protocolo ERAS en cirugía colorrectal concluyeron que con la aplicación del Protocolo ERAS se reduce la estadía hospitalaria 2,50 a 2,94 días y se reduce la tasa de complicaciones entre 47 y $52 \%$, de manera estadísticamente significativa. Lo anterior, además, sin aumentar el número de readmisiones o mortalidad postoperatoria y optimizando el uso de los recursos económicos para los sistemas de salud con reducción en costos que van entre 980 y 2.240 dólares americanos $^{42-44}$. Estos excelentes resultados han promovido que se desarrollen protocolos ERAS para otros tipos de cirugías como: digestiva alta, ginecológica, urológica ${ }^{14-18}$, etc.

En Chile, el equipo de cirugía coloproctológica del Hospital Militar ha implementado los aspectos anestésicos y quirúrgicos del Protocolo ERAS para resección de colon por vía abierta, publicando buenos resultados en $2009^{45}$. En este estudio, Espíndola y colaboradores demostraron que utilizando un protocolo similar al ERAS (sin carga de hidratos de carbono preoperatorios) era posible reducir la estadía hospitalaria de más de 7 días a 4-5 días postoperatorios. Además, 97\% de los enfermos que se operó con este protocolo similar a ERAS presentó tránsito intestinal para gases en las primeras $48 \mathrm{~h}$ y $87 \%$ defecación entre el $2^{\text {do }}$ y $3^{\text {er }}$ día, mientras que con cuidados tradicionales, $73 \%$ de los enfermos presentaban defecación entre el $6^{\text {to }}$ y $7^{\text {mo }}$ día postoperatorio. Actualmente, en el Hospital Clínico de la Universidad de Chile, se ha aplicado el Protocolo ERAS en cirugía colorrectal por vía abierta y laparoscópica, incluyendo las intervenciones nutricionales de prevención del APP con carga de hidratos de carbono (maltodextrina al $12,5 \%$ ) vía oral 2-3 h antes de la cirugía y posterior realimentación precoz desde el primer día postoperatorio, con lo cual se ha logrado una disminución de 1,5 días de estadía hospitalaria, sin aumentar las complicaciones, reoperaciones ni mortalidad.

A pesar de que muchos estudios han demostrado que aplicar el Protocolo ERAS, logra acelerar la recuperación del paciente y disminuir la estadía hospitalaria, en comparación con el uso de cuidados tradicionales, aún la implementación y buena adherencia a este protocolo es baja a nivel mundial ${ }^{46}$, sin embargo, a la luz de la evidencia científica actual, debemos continuar realizando esfuerzos por derrumbar paradigmas aprendidos y practicados de manera tradicional, pero carentes de sustento científico, y cambiar así el manejo nutricional perioperatorio de nuestros pacientes, con el fin de lograr la mejor recuperación postoperatoria posible.

\section{Referencias}

1. Cullen KA, Hall MJ, Golosinskiy A. Ambulatory Surgery in the United States 2006. National Health Statistics Reports. No 11. Revised. Hyattsville, MD: National Center for Health Statistics, 2009.

2. Csendes A, González G. Cirugías digestivas más frecuentes en Chile, excluyendo colorrectal. Rev Chilena Cirugía 2008; 60(5): 379-86.

3. Superintendencia de Salud de Chile [internet]. Santiago: Problema de salud AUGE. Disponible en: http://www. supersalud.gob.cl/568/w3-propertyvalue-527.html

4. Masoomi H, Buchberg B, Dang P, Carmichael JC, Mills $\mathrm{S}$, Stamos MJ. Outcomes of right vs. left colectomy for colon cancer. J Gastrointest Surg 2011; 15 (11): 2023-8.

5. López F, Zárate A, León F, García-Huidobro MA, Bellolio F, Pinedo G, et al. Resultados preliminares de la cirugía laparoscópica del cáncer colorrectal. Rev Chilena Cirugía 2006; 58 (2): 106-13.

6. Gellona J, Bellolio F, Molina ME, Miguieles R, Urrejola G, Zúñiga A. Cáncer de recto. Análisis de las complicaciones post operatorias y mortalidad de la resección radical en una serie de 10 años. Rev Chilena Cirugía 2013; 65 (3): 242-8. 
7. Braghetto I, Csendes A, Cardemil G, Burdiles P, Rodríguez A, Guerra JF. Complicaciones de la esofagectomía: diagnóstico, mecanismo fisiopatológico, prevención y manejo. Rev Chilena Cirugía 2002; 54(5): 451-63.

8. Wilmore DW. From Cuthbertson to Fast-Track Surgery: 70 Years of Progress in Reducing Stress in Surgical Patients. Ann Surg 2002; 236 (5): 643-8.

9. Nygren J. The metabolic effects of fasting and surgery. Best Pract Res Clin Anaesthesiol 2006; 20: 429-38.

10. Thorell A, Nygren J, Ljungqvist O. Insulin resistance: a marker of surgical stress. Curr Opin Clin Nutr Metab Care 1999; 2: 69-78.

11. Van den Berghe G, Wouters PJ, Bouillon R, Weekers F, Verwaest C, Schetz M, et al. Outcome benefit of intensive insulin therapy in the critically ill: insulin dose versus glycemic control. Crit Care Med 2003; 31: 359-66.

12. Soop M, Nygren J, Thorell A, Ljungqvist O. Stress-induced insulin resistance: recent developments. Curr Opin Clin Nutr Metab Care 2007; 10: 181-86.

13. Fearon $\mathrm{KCH}$, Ljungqvist $\mathrm{O}$, Von Meyenfeldt M, Revhaug A, Dejong CHC, Lassen K, et al. Enhanced recovery after surgery: A consensus review of clinical care for patients undergoing colonic resection. Clin Nutr 2005; 24: 466-77.

14. Gustafsson UO, Scott MJ, Schwenk W, Demartines N, Roulin D, Francis N, et al. Enhanced Recovery After Surgery (ERAS) Society, for Perioperative Care; European Society for Clinical Nutrition and Metabolism (ESPEN); International Association for Surgical Metabolism and Nutrition (IASMEN). Guidelines for perioperative care in elective colonic surgery: Enhanced Recovery After Surgery (ERAS( $\left.{ }^{\circledR}\right)$ ) Society recommendations. World J Surg 2013; 37 (2): 259-84.

15. Nygren J, Thacker J, Carli F, Fearon KC, Norderval S, Lobo DN, et al. Enhanced Recovery After Surgery (ERAS) Society, for Perioperative Care; European Society for Clinical Nutrition and Metabolism (ESPEN); International Association for Surgical Metabolism and Nutrition (IASMEN). Guidelines for perioperative care in elective rectal/pelvic surgery: Enhanced Recovery After Surgery (ERAS $\left.\left({ }^{\circledR}\right)\right)$ Society recommendations. World J Surg 2013; 37 (2): 285-305.

16. Mortensen K, Nilsson M, Slim K, Schäfer M, Mariette C, Braga M, et al. Enhanced Recovery After Surgery $\left(\right.$ ERAS $\left.^{\circledR}\right)$ Group. Consensus guidelines for enhanced recovery after gastrectomy: Enhanced Recovery After Surgery (ERAS ${ }^{\circledR}$ ) Society recommendations. Br J Surg 2014; 101 (10): 1209-29.

17. Lassen K, Coolsen MM, Slim K, Carli F, de Aguilar-Nascimento JE, Schäfer M, et al. Enhanced Recovery After Surgery (ERAS) Society, for Perioperative
Care; European Society for Clinical Nutrition and Metabolism (ESPEN); International Association for Surgical Metabolism and Nutrition (IASMEN). Guidelines for perioperative care for pancreaticoduodenectomy: Enhanced Recovery After Surgery (ERAS ${ }^{\circledR}$ ) Society recommendations. World J Surg 2013; 37 (2): 240-58.

18. Cerantola Y, Valerio M, Persson B, Jichlinski P, Ljungqvist $\mathrm{O}$, Hubner $\mathrm{M}$, et al. Guidelines for perioperative care after radical cystectomy for bladder cancer: Enhanced Recovery After Surgery (ERAS $\left.{ }^{\circledR}\right)$ society recommendations. Clin Nutr 2013; 32 (6): 879-87.

19. Ljungqvist O. Modulating postoperative insulin resistance by preoperative carbohydrate loading. Best Pract Res Clin Anaesthesiol 2009; 23 (4): 401-9.

20. Ljungqvist O, Soreide, E. Preoperative fasting. Br J Surg 2003; 90 (4): 400-6.

21. Brady M, Kinn S, Stuart P. Preoperative fasting for adults to prevent perioperative complications. Cochrane Database Syst Rev 2003; (4): CD004423.

22. Hausel J, Nygren J, Lagerkranser M, Hellström PM, Hammarqvist F, Almström C, et al. A carbohydrate-rich drink reduces preoperative discomfort in elective surgery patients. Anesth Analg 2001; 93 (5): 1344-50.

23. Nygren J, Soop M, Thorell A, Efendic S, Nair KS, Ljungqvist O. Preoperative oral carbohydrate administration reduces postoperative insulin resistance. Clin Nutr 1998; 17 (2): 65-71.

24. Svanfeldt M, Thorell A, Hausel J, Soop M, Rooyackers $\mathrm{O}$, Nygren J, et al. Randomized clinical trial of the effect of preoperative oral carbohydrate treatment on postoperative whole-body protein and glucose kinetics. Br J Surg 2007; 94 (11): 1342-50.

25. Henriksen MG, Hessov I, Dela F, Hansen HV, Haraldsted V, Rodt SA. Effects of preoperative oral carbohydrates and peptides on postoperative endocrine response, mobilization, nutrition and muscle function in abdominal surgery. Acta Anaesthesiol Scand 2003; 47 (2): 191-9.

26. Yuill KA, Richardson RA, Davidson HI, Garden OJ, Parks RW. The administration of an oral carbohydrate-containing fluid prior to major elective uppergastrointestinal surgery preserves skeletal muscle mass postoperatively - a randomised clinical trial. Clin Nutr 2005; 24 (1): 32-7.

27. Practice guidelines for preoperative fasting and the use of pharmacologic agents to reduce the risk of pulmonary aspiration: application to healthy patients undergoing elective procedures: a report by the American Society of Anesthesiologist Task Force on Preoperative Fasting. Anesthesiology 1999; 90 (3): 896-905. 
28. Soreide E, Eriksson LI, Hirlekar G, Eriksson H, Henneberg SW, Sandin R, et al. Pre-operative fasting guidelines: an update. Acta Anaesthesiol Scand 2005; 49 (8): 1041-7.

29. Smith I, Kranke P, Murat I, Smith A, O'Sullivan G, Soreide E, et al. Perioperative fasting in adults and children: guidelines from the European Society of Anaesthesiology. Eur J Anaesthesiol 2011; 28 (8): 556-69.

30. Breuer JP, von Dossow V, von Heymann C, Griesbach M, von Schickfus M, Mackh E, et al. Preoperative oral carbohydrate administration to ASA III-IV patients undergoing elective cardiac surgery. Anesth Analg 2006; 103: 1099-108.

31. Gustafsson UO, Nygren J, Thorell A, Soop M, Hellström $\mathrm{PM}$, Ljungqvist $\mathrm{O}$, et al. Pre-operative carbohydrate loading may be used in type 2 diabetes patients. Acta Anaesthesiol Scand 2008; 52 (7): 946-51.

32. Andersen HK, Lewis SJ, Thomas S. Early enteral nutrition within $24 \mathrm{~h}$ of colorectal surgery versus later commencement of feeding for postoperative complications. Cochrane Database Syst Rev 2006; (4): CD004080.

33. Lewis SJ, Andersen HK, Thomas S. Early enteral nutrition within $24 \mathrm{~h}$ of intestinal surgery versus later commencement of feeding: a systematic review and meta-analysis. J Gastrointest Surg 2009; 3: 569-75.

34. Osland E, Yunus RM, Khan S, Memon MA. Early Versus Traditional Postoperative Feeding in Patients Undergoing Resectional Gastrointestinal Surgery: A Meta-Analysis. J Parenter Enteral Nutr 2011; 35: 47387.

35. Uden $\mathrm{P}$, Blomquist $\mathrm{P}$, Jiborn $\mathrm{H}$, Zederfeldt B. Impact of longterm relative bowel rest on conditions for colonic surgery. Am J Surg 1988; 156: 381-5.

36. Moss G, Greenstein A, Levy S, Bierenbaum A. Maintenance of GI function after bowel surgery and immediate enteral full nutrition. I. Doubling of canine colorectal anastomotic bursting pressure and intestinal wound mature collagen content. JPEN J Parenter Enteral Nutr 1980; 4: 535-8.

37. Soop M, Carlson GL, Hopkinson J, Clarke S, Thorell A, Nygren J, et al. Randomized clinical trial of the effects of immediate enteral nutrition on metabolic responses to major colorectal surgery in an enhanced recovery protocol. Br J Surg 2004; 91: 1138-45.

38. Miller TE, Thacker JK, White WD, Mantyh C, Migaly J, Jin J, et al. Reduced length of hospital stay in colorectal surgery after implementation of an enhanced recovery protocol. Anesth Analg 2014; 118 (5): 1052-61.

39. Lobo DN, Bostock KA, Neal KR, Perkins AC, Rowlands BJ, Allison SP. Effect of salt and water balance on recovery of gastrointestinal function after elective colonic resection: a randomised controlled trial. Lancet 2002; 359 (9320): 1812-8.

40. Marimuthu K, Varadhan KK, Ljungqvist O, Lobo DN. A meta-analysis of the effect of combinations of immune modulating nutrients on outcome in patients undergoing major open gastrointestinal surgery. Ann Surg 2012; 255 (6): 1060-8.

41. Wind J, Polle SW, Fung Kon Jin PHP, Dejong CHC, vonMeyenfeldt MF, Ubbink DT, et al. Systematic review of enhanced recovery programmes in colonic surgery. $\mathrm{Br}$ J Surg 2006; 93: 800-9.

42. Varadhan KK, Neal KR, Dejong CH, Fearon KC, Ljungqvist $\mathrm{O}$, Lobo DN. The enhanced recovery after surgery (ERAS) pathway for patients undergoing major elective open colorectal surgery: a meta-analysis of randomized controlled trials. Clin Nutr 2010; 29 (4): 434-40.

43. Spanjersberg WR, Reurings J, Keus F, van Laarhoven CJ. Fast track surgery versus conventional recovery strategies for colorectal surgery. Cochrane Database Syst Rev 2011; (2): CD00763.

44. Adamina M, Kehlet H, Tomlinson GA, Senagore AJ, Delaney CP. Enhanced recovery pathways optimize health outcomes and resource utilization: a meta-analysis of randomized controlled trials in colorectal surgery. Surgery 2011; 149 (6): 830-40.

45. Espíndola L. Cirugía de colon abierta con "Fast Track" o recuperación acelerada. Rev Chilena Cirugía 2009; 2: 158-67.

46. Roiga JV, Rodríguez-Carrillo R, García-Armengol J, Villalba FL, Salvador A, Sancho C, et al. Rehabilitación mutimodal en cirugía colorrectal. Sobre la resistencia al cambio en cirugía y las demandas de la sociedad. Cir Esp 2007; 81: 307-15. 